\title{
ESTADO DA ARTE DA PRODUÇÃO CIENTÍFICA SOBRE INDICADORES E ÍNDICES DE INOVAÇÃO
}

\section{Rafael de Moura Speroni}

Doutorando em Engenharia e Gestão do Conhecimento pela Universidade Federal de Santa Catarina UFSC

Professor de Informática no Instituto Federal Catarinense - IFC

rafaelsperoni@gmail.com (Brasil)

\section{Gertrudes Aparecida Dandolini}

Doutora em Engenharia de Produção pela Universidade Federal de Santa Catarina - UFSC

Professora da Universidade Federal de Santa Catarina - UFSC

gtude@egc.ufsc.br (Brasil)

\section{João Artur Souza}

Doutor em Engenharia de Produção pela Universidade Federal de Santa Catarina - UFSC

Professora da Universidade Federal de Santa Catarina - UFSC

jartur@egc.ufsc.br (Brasil)

\section{Fernando A. Ostuni Gauthier}

Doutor em Engenharia de Produção pela Universidade Federal de Santa Catarina - UFSC

Professora da Universidade Federal de Santa Catarina - UFSC

gauthier@egc.ufsc.br (Brasil)

\section{RESUMO}

A gestão da inovação mostra-se um desafio para as organizações na busca pela competitividade. Ao implementar um processo de gestão, tão importante quanto explicitar o modelo estratégico da inovação, é a capacidade de mensuração do que se deseja gerenciar. $\mathrm{O}$ uso de indicadores e índices de inovação permite, portanto, a verificação do desempenho de organizações ou regiões em separado ou em comparação com outras. A definição de indicadores de inovação não é, portanto, uma tarefa trivial, e diversas são ferramentas utilizadas, tais como estatísticas de patentes e P\&D. Este trabalho apresenta uma revisão bibliométrica com o propósito mapear a produção científica relativa ao tema indicadores de inovação, identificando autores, obras e fontes de publicação mais relevantes. Para isso, foram analisados 107 trabalhos científicos que abordam o referido tema, escritos por 209 autores pertencentes a 115 instituições de 35 países diferentes. Os resultados mostram que a primeira publicação encontrada data do ano de 1973, mas o número de publicações mostra uma tendência de crescimento com o tempo e predominam as publicações em journals e a Europa é a região com maior número de autores, seguida da China, Brasil e Estados Unidos.

Palavras-chave: Indicadores de Inovação; Índices de Inovação; Inovação; Bibliometria; Pesquisa bibliográfica. 


\section{INTRODUÇÃO}

O termo "indicadores de inovação" tem considerável importância na área acadêmica e organizacional no que tange à temática da inovação. As empresas buscam a inovação, pois conforme afirma Bessant and Tidd (2009), se não mudam seus produtos ou serviços, a forma como os cria e oferta, correm o risco de serem superadas por outras que os façam.

Nesse sentido, tão importante quanto desenvolver a capacidade de fazer inovação, é a capacidade de mensuração desse processo, uma vez que disto depende a competitividade de uma empresa. Para Quintane, Casselman, Reiche, and Nylund (2011), compreender a capacidade de inovação das organizações é, portanto, fundamental para os gestores, e isso exige uma definição precisa do que constitui uma inovação.

A inovação é complexa, e não há um consenso sobre qual indicador deve ser usado para medila (Makkonen \& van der Have, 2013). Além disso, o tipo de variável escolhida pode afetar as conclusões de pesquisa, ou as informações nas quais os decisores políticos baseiam suas decisões. Zabala-Iturriagagoitia, Jiménez-Sáez, Castro-Martínez, and Gutiérrez-Gracia (2007) afirmam que, embora muitos estudos tenham proposto metodologias para medir a capacidade de inovação, ainda havia muito trabalho a ser feito, uma vez que os estudos existentes, bem como as estatísticas disponíveis não levavam em conta os aspectos institucionais, interações, acordos de cooperação, entre outros, que são considerados elementos cruciais de um Sistema de Inovação.

Medidas comumente aplicadas, por serem consideradas proxies para a inovação, são as estatísticas de Pesquisa e Desenvolvimento (P\&D) e de patentes, sob o argumento de que o aumento nos esforços de $\mathrm{P} \& \mathrm{D}$ em uma região resulta em uma taxa maior de invenção, assim como o de que um número maior de patentes leva a mais inovações. Embora P\&D e Patentes sejam considerados indicadores válidos para medir a inovação, tais relações lineares podem ser ilusórias, visto que estes indicadores apresentam fragilidades (Makkonen \& van der Have, 2013; Nelson, 2009; Schmoch \& Gauch, 2009).

Embora as estatísticas de P\&D estejam associadas à inovação, nem todos os esforços de P\&D estão necessariamente relacionados a resultados de inovação comercializados ou de sucesso (Makkonen \& van der Have, 2013; Nelson, 2009). Há, ainda, o entendimento de que P\&D pode ser considerado apenas um fator de entrada (input) da inovação (Kleinknecht, Van Montfort, \& Brouwer, 2002; Ratanawaraha \& Polenske, 2007). De forma semelhante, nem todas as invenções patenteadas tornam-se inovações, e o conteúdo e valor econômico das patentes pode diferir largamente entre elas.

Revista de Administração e Inovação, São Paulo, v. 12, n.4 p. 49-75, out./dez. 2015. 
Rafael de Moura Speroni, Gertrudes Aparecida Dandolini, João Artur Souza \& Fernando A. Ostuni Gauthier

Além disso, alguns setores tecnológicos geram mais patentes do que outros, e o tamanho das empresas também influencia na propensão de patentear (Grupp \& Schubert, 2010; Kleinknecht et al., 2002; Nelson, 2009).

Outra importante forma de mensurar a inovação é a quantificação direta dos resultados da inovação (Makkonen \& Have, 2013). Tais indicadores são comumente obtidos por meio de questionários aplicados a empresas, por meio dos quais são explorados os fatores que influenciam seu comportamento inovador, suas estratégias, incentivos e barreiras para a inovação, o escopo das várias atividades de inovação e, sobretudo, os resultados e efeitos da inovação. Esta abordagem é conhecida como subject-approach, uma vez que as próprias empresas respondentes são o alvo da pesquisa (OECD, 2005). Embora seja uma abordagem largamente aplicada, citam-se como possíveis deficiências: a falta de objetividade nas respostas; a falta de entendimento do respondente em relação ao que é inovação; a existência de respostas estimadas; as baixas taxas de resposta; a heterogeneidade de valor econômico das inovações reportadas; o custo de produção das medidas de inovação; e a limitada disponibilidade de dados (Kleinknecht et al., 2002; Ratanawaraha \& Polenske, 2007).

A complexidade da inovação é, portanto, refletida na dificuldade de mensurá-la. Como o entendimento de Schumpeter sobre a inovação não é dado em termos de formalismos matemáticos, tornou-se uma prática comum a combinação de diversos indicadores de ciência, tecnologia e inovação para formar números agregados ou índices, normalmente apresentados na forma de um ranking ou normalizado em um intervalo fechado (Grupp \& Schubert, 2010).

Um indicador composto como é uma métrica única de valor real que é derivada de algum método de agregação de um conjunto de componentes indicadores, segundo um modelo do domínio da política que se deseja mensurar (Grupp \& Schubert, 2010; Saisana, Saltelli, \& Tarantola, 2005). Diversas iniciativas tratam da criação de indicadores de inovação compostos, possibilitando estudos comparativos entre diferentes países e regiões (Cornell University, INSEAD, \& WIPO, 2013; IBGE, 2010; OECD, 2005).

Este artigo objetiva mapear a produção científica, identificando as características dos trabalhos científicos que abordam os temas "indicadores de inovação" e "índices de inovação". Conforme afirma Pizzani (2012), a realização desse tipo de levantamento da literatura contribui para sua melhor compreensão, aponta as iniciativas que estão sendo desenvolvidas, novas linhas de pesquisas, além de apresentar os mais representativos autores e grupos de pesquisas que têm desenvolvido estudos associados à temática.

O documento está organizado em quatro seções. A seção 2 apresenta os procedimentos metodológicos utilizados para o desenvolvimento da pesquisa bibliométrica. A seção 3 apresenta a

Revista de Administração e Inovação, São Paulo, v. 12, n.4 p. 49-75, out./dez. 2015. 
discussão dos principais resultados. Finalmente, na seção 4, são descritas as considerações finais do artigo, seguidas pelo referencial teórico.

\section{PROCEDIMENTOS METODOLÓGICOS}

A análise bibliométrica e de redes sociais no meio acadêmico desempenha papel fundamental para o fomento, a disseminação e a socialização do conhecimento científico. É fato que o número de estudos bibliométricos e sociométricos na exploração de acervos de periódicos nacionais e internacionais tem aumentado significativamente em várias áreas do conhecimento (Ribeiro, Cirani, \& Freitas, 2013).

A pesquisa aqui apresentada foi desenvolvida combinando técnicas bibliométricas e bibliográficas (Araújo \& Alvarenga, 2011; Kobashi \& Santos, 2006; Macias-Chapula, 1998). Em uma primeira etapa, foi realizada uma busca sistêmica da literatura, constituída da coleta, filtragem e padronização dos dados. A segunda etapa consiste de uma análise descritiva composta pela análise e seleção dos dados coletados, culminando na elaboração do documento final. As subseções seguintes descrevem os procedimentos adotados em cada uma das etapas de confecção do artigo.

\subsection{Etapa 1 - Definição dos termos de busca}

Para a realização das buscas bibliográficas nas bases científicas foi utilizado os termos em inglês "innovation index*" e "innovation indicator*”. O símbolo “*” visa incluir nas buscas os resultados com os termos no plural, como "innovation indexes" e "innovation indicators".

\subsection{Etapa 2 - Consulta nas bases de dados}

As buscas de onde resultam os dados aqui descritos foram feitas em quatro bases de dados científicos internacionais: Web of Science ${ }^{1}$ (WoS), Scopus $^{2}$, EBSCO $^{3}$ e IEEE $^{4}$.

Cada uma das bases pesquisadas apresenta características próprias de estruturação e indexação dos termos de pesquisa, o que requer estratégias de busca diferentes para cada uma das bases. A

\footnotetext{
${ }^{1}$ http://apps.webofknowledge.com

2 http://www.scopus.com/home.url

${ }^{3}$ http://search.ebscohost.com

${ }^{4}$ http://ieeexplore.ieee.org/Xplore/guesthome.jsp
}

Revista de Administração e Inovação, São Paulo, v. 12, n.4 p. 49-75, out./dez. 2015. 
Rafael de Moura Speroni, Gertrudes Aparecida Dandolini, João Artur Souza \& Fernando A. Ostuni Gauthier pesquisa foi realizada em outubro de 2013, e, além da restrição resultante da escolha do campo de busca, os artigos foram restritos aos idiomas inglês, português e espanhol, pertencentes a revistas e congressos, e classificados nas áreas de engenharia, administração e ciências sociais.

\subsection{Etapa 3- Exportação dos registros}

As listas de resultados das buscas bibliográficas nas bases científicas foram exportadas e carregadas em uma biblioteca no software de gestão de referências bibliográficas EndNote ${ }^{5}$. Dessa forma, os dados foram integrados em um conjunto único, para possibilitar a padronização dos metadados.

\subsection{Etapa 4 - Aplicação de critérios de seleção}

Nesta etapa do desenvolvimento foram aplicados os seguintes critérios de seleção: 1) exclusão de artigos sem autoria; 2) exclusão de artigos duplicados, ou seja, artigos que estavam indexados em mais de uma base de dados; 3) retirada dos artigos que estavam fora do contexto deste estudo; e 4) retirada de artigos no qual os textos completos não estão disponíveis gratuitamente.

\subsection{Etapa 5 - Padronização dos dados}

Em função do uso de dados obtidos de diferentes bases científicas, havia diferenças na forma como os dados eram disponibilizados em cada uma delas. Dessa forma, buscou-se a padronização dos mesmos, a fim de que não houvesse ambiguidades nos nomes de autores, bem como houve a complementação de informações referentes às instituições às quais os autores são vinculados, e sua localização.

Os dados resultantes da padronização no Endnote foram posteriormente cadastrados em uma base de dados usando o Microsoft Access, no qual foi feito o preenchimento das informações faltantes, como informações dos autores (sobrenome, nome, afiliação e localização da instituição), dos artigos (ano de publicação, idioma, tipo de documento, fonte de publicado, resumo e principais temáticas), das referências citadas por cada artigo (autor, ano, título do trabalho) e as palavras-chave.

${ }^{5}$ http://www.endnote.com 


\subsection{Etapa 6 - Análise dos dados}

Após a etapa de padronização dos dados ser concluída, foi possível passar à fase de análise bibliométrica dos dados. Foram analisadas as quantidades de publicações por ano, os tipos de fonte de publicação, vínculos e países de origem dos autores, ocorrência de palavras-chave, e trabalhos referenciados pelos artigos.

O objetivo desta fase de análise é traçar um cenário da localização e caracterização dos possíveis grupos de pesquisas que desenvolvem trabalhos sobre o tema de indicadores de inovação. Desejou-se ainda, nesta etapa, a identificação das mais significativas referências bibliográficas utilizadas pelos autores nos trabalhos selecionados.

Posteriormente, pela leitura dos abstracts dos artigos selecionados, buscou-se a classificação dos mesmos em grupos de diferentes temáticas. A análise das palavras-chave com maior ocorrência permitiu a identificação de termos que nortearam a definição dos grupos temáticos.

\subsection{Etapa 7 - Elaboração do documento final}

Este documento contém descrições e os resultados das análises, e está organizado na forma de um artigo científico. Nas próximas seções são apresentados os resultados obtidos por meio do desenvolvimento de cada uma das etapas aqui descritas.

\section{RESULTADOS}

Esta seção apresenta os resultados das análises e sínteses das informações bibliométricas, selecionadas a partir da busca bibliográfica, padronização e análise dos dados.

\subsection{Dados bibliométricos gerais sobre as pesquisas em Indicadores de Inovação}

A busca bibliométrica nas bases de dados Web of Science, Scopus, EBSCO e IEEE apresentou inicialmente um total de 504 registros. Após a aplicação dos diversos filtros, restou um total de 107 
trabalhos que serão analisados no decorrer desta pesquisa. A Tabela 1 ilustra o processo de seleção dos artigos para a análise final.

Tabela 1 - Número de publicações selecionadas nas bases de dados

\begin{tabular}{cccccc}
\hline $\begin{array}{c}\text { Bases } \\
\text { de } \\
\text { Dados }\end{array}$ & $\begin{array}{c}\text { Publicações } \\
\text { Localizadas }\end{array}$ & $\begin{array}{c}\text { Exclusão } \\
\text { das } \\
\text { Publicações } \\
\text { Repetidas }\end{array}$ & $\begin{array}{c}\text { Exclusão de } \\
\text { Publicações } \\
\text { sem Autoria } \\
\text { Disponível }\end{array}$ & $\begin{array}{c}\text { Exclusão de } \\
\text { Publicações sem Texto } \\
\text { Completo Disponível } \\
\text { ou Fora de Contexto }\end{array}$ & $\begin{array}{c}\text { Total de } \\
\text { Publicações } \\
\text { Selecionadas }\end{array}$ \\
\hline WoS & 71 & 50 & 1 & 14 & 6 \\
Scopus & 202 & 9 & 8 & 102 & 83 \\
Ebsco & 217 & 78 & 51 & 71 & 17 \\
IEEE & 14 & 12 & 0 & 1 & 1 \\
Total & $\mathbf{5 0 4}$ & $\mathbf{1 4 9}$ & $\mathbf{6 0}$ & $\mathbf{1 8 8}$ & $\mathbf{1 0 7}$ \\
\hline \multicolumn{5}{c}{ Fonte: Elaborado pelos autores }
\end{tabular}

Entre os 107 trabalhos selecionados, dois são working papers, 15 são artigos de congressos e 89 indexados em periódicos científicos. Esses trabalhos foram escritos por 209 autores, vinculados a 115 instituições de 35 países diferentes e publicados em 73 fontes diferentes. Os autores utilizaram 300 palavras-chave para indexar seus trabalhos e 2.583 referências (média de 24 referências citadas por publicação). 
Tabela 2 sintetiza os dados bibliométricos gerais da pesquisa. 
Tabela 2 -Dados bibliométricos gerais das publicações selecionadas nas bases de dados

\begin{tabular}{lc}
\hline Dados bibliométricos & Frequência \\
\hline Publicações & 107 \\
Fontes de publicações & 73 \\
Autores & 209 \\
Instituições & 115 \\
Países & 35 \\
Palavras-chave & 300 \\
Referências citadas & 2.583 \\
\hline
\end{tabular}

Fonte: Elaborado pelos autores

Na sequência serão descritas as análises resultantes de cada um dos dados bibliométricos apresentados na Tabela 2.

\subsection{Publicações por ano}

A série histórica aponta que o maior número de artigos, conforme demonstra o gráfico da Erro! Fonte de referência não encontrada., foi no ano de 2012. Nesse ano, 19 artigos foram publicados, sendo 16 em journals e três em conferências ou workshops.

Figura 1 - Total de artigos por ano

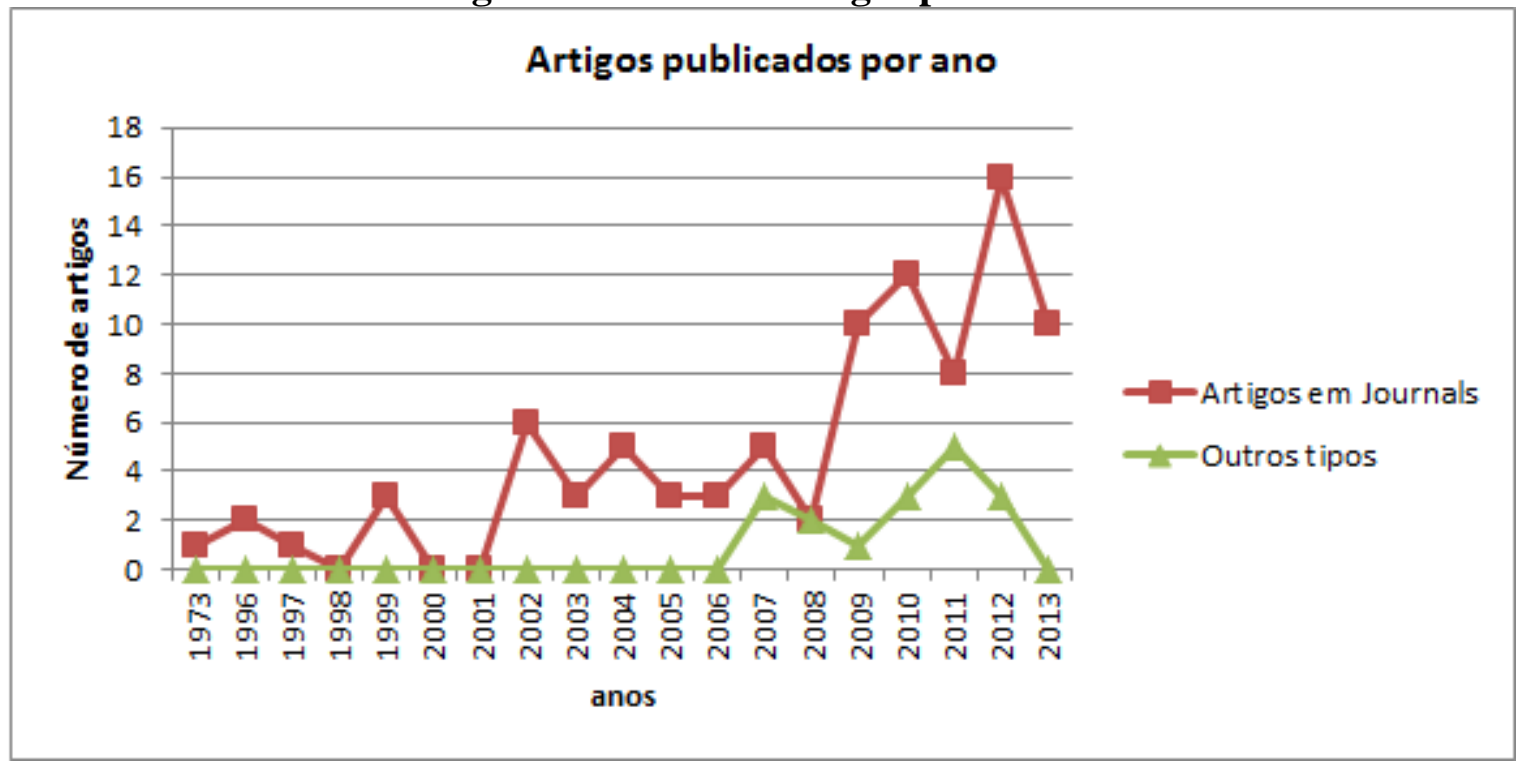

Fonte: Elaborado pelos autores

Observa-se que, o assunto "Indicadores de Inovação" não é um tema propriamente novo, e a primeira publicação identificada nas bases de dados pesquisadas, que trata do assunto, é de 1973. Nesse ano, foi identificado o trabalho An Innovation Index Based on Factor Analysis (Blackman Jr, Seligman, \& Sogliero, 1973), publicado no Journal Technological Forecasting and Social Change. O 
Estado da arte da produção científica sobre indicadores e índices de inovação

trabalho trata da construção de um índice de inovação que indica a tendência relativa para a inovação em setores industriais, levando em consideração variáveis de entrada que refletem a extensão de alocação de recursos para o desenvolvimento de novos produtos e inovação em processos.

Não foram identificadas publicações no período entre os anos de 1974 e 1995. O número de publicações sobre o assunto apresenta tendência de crescimento a partir de 1996, excetuando-se os anos de 1998, 2000 e 2001 que não contam com publicações. A partir do ano de 2009, tem-se sempre mais de dez artigos por ano, e no ano de 2013, embora os dados sejam parciais, o número de publicações já é igual a dez.

\subsection{Principais fontes de publicações}

Os artigos foram publicados em 73 fontes de publicações diferentes, sendo que, destas, 51 são journals. As cinco fontes de publicação mais utilizadas pelos autores estão apresentadas na

Tabela 3 e descritas em seguida.

Tabela 3 -Fontes de publicação com maior ocorrência

\begin{tabular}{cccccc}
\hline Fonte de publicação & Qtd & ISSN & Tipo & $\begin{array}{c}\text { Fator de } \\
\text { Impacto (JRC) }\end{array}$ & Editora \\
\hline Scientometrics & 8 & $\begin{array}{r}0138- \\
9130\end{array}$ & Journal & 2.133 & Springer \\
\hline Research Policy & 7 & $\begin{array}{r}0048- \\
7333\end{array}$ & Journal & 2.850 & Elsevier \\
\hline Research Evaluation & 5 & $\begin{array}{r}0958- \\
2029\end{array}$ & Journal & 1.074 & Oxford Journals \\
\hline Technovation & 5 & $\begin{array}{r}0166- \\
4972\end{array}$ & Journal & 3.177 & Elsevier \\
\hline $\begin{array}{c}\text { Technological } \\
\text { Forecasting and Social } \\
\text { Change }\end{array}$ & 4 & $\begin{array}{c}0040- \\
1625\end{array}$ & Journal & 2.106 & Elsevier \\
\hline
\end{tabular}

Fonte: Elaborado pelos autores

O periódico científico com maior número de publicações é o Scientometrics, com oito trabalhos, e tem ênfase em investigações em que o desenvolvimento e os mecanismos da ciência são estudados por meio de métodos matemáticos e estatísticos. O segundo periódico com maior número de publicações é o Research Policy, que conta com sete artigos, e dedica-se a examinar empírica e teoricamente as interações entre inovação, tecnologia ou pesquisa, e processos organizacionais econômicos, sociais e políticos.

Com cinco publicações em cada, citam-se os periódicos Research Evaluation e Technovation. $\mathrm{O}$ primeiro trata-se de uma revista interdisciplinar que abrange os métodos emergentes, experiências e 
Rafael de Moura Speroni, Gertrudes Aparecida Dandolini, João Artur Souza \& Fernando A. Ostuni Gauthier

lições para pesquisas em avaliação e estimativas. Já o segundo é uma revista que abrange todas as facetas do processo de inovação tecnológica, além de estudos de caso que ilustram como a inovação ocorre, juntamente com comentários e análises de política governamental e industrial que inibem ou estimulam a inovação tecnológica.

Por fim, a revista Technological Forecasting and Social Change, com quatro publicações, é descrita como um fórum para aqueles que desejam lidar diretamente com a metodologia e prática de previsão tecnológica e estudos futuros como ferramentas de planejamento que relacionam fatores sociais, ambientais e tecnológicos.

\subsection{Principais autores, instituições e países}

As publicações analisadas neste trabalho foram escritas por 209 autores que estão afiliados a 115 instituições. O mapa da Erro! Fonte de referência não encontrada. ilustra a localização dessas instituições, na forma de um mapa que indica a intensidade de ocorrências, onde a cor verde indica uma menor intensidade e a cor vermelha indica uma maior intensidade. Pelo mapa, percebe-se que a Europa (França com 14, Alemanha com 12, Espanha com 12, Reino Unido com nove e Holanda com oito pesquisadores), a China (com 23 pesquisadores), o Brasil (com 22 pesquisadores) e os Estados Unidos (com 20 pesquisadores) são os locais com maior número de publicações no conjunto em estudo. 


\section{Figura 2 -Mapa com a distribuição das instituições às quais os autores pertencem}

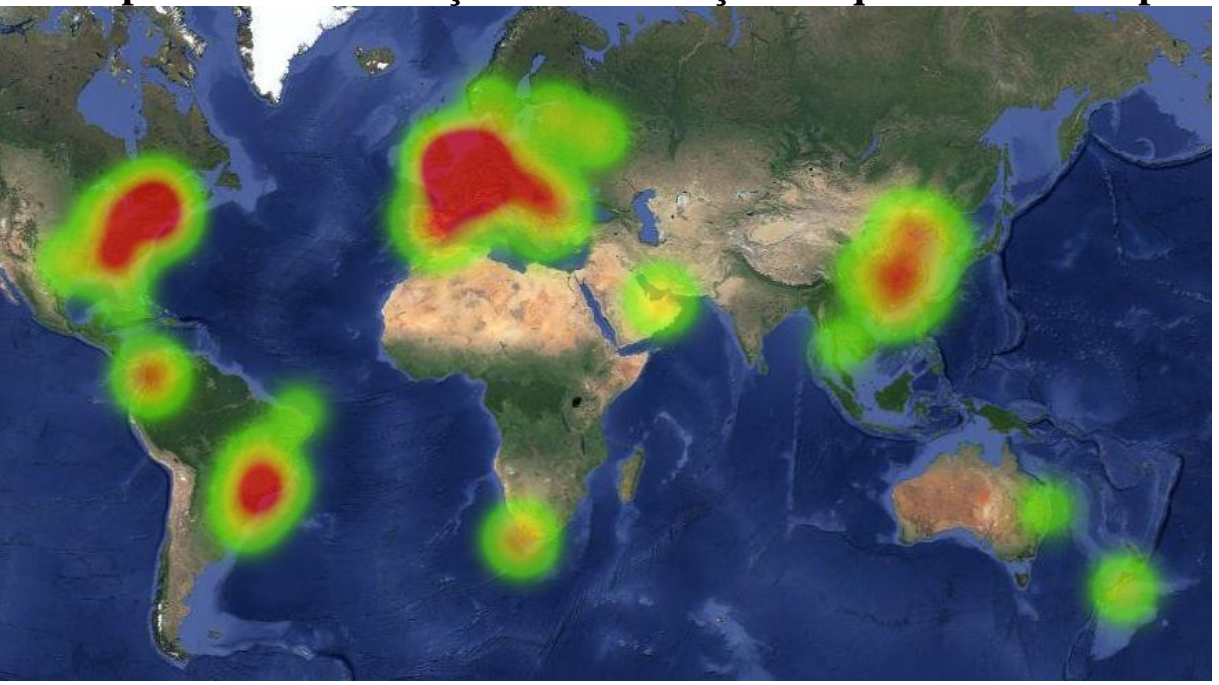

Fonte: Elaborado pelos autores

Embora haja maior intensidade de ocorrências de publicações em algumas regiões, foram observados poucos autores com mais de uma publicação sobre indicadores de inovação. A

Tabela 4 apresenta os autores com maior número de publicação.

Tabela 4 -Autores com maior número de publicações

\begin{tabular}{|c|c|c|c|c|c|}
\hline \multirow[b]{2}{*}{ Autor } & \multicolumn{2}{|c|}{ Publicações } & \multirow[b]{2}{*}{ Vínculo Institucional } & \multirow[b]{2}{*}{ Cidade } & \multirow[b]{2}{*}{ País } \\
\hline & Total & $\begin{array}{c}1^{\circ} \\
\text { Autor }\end{array}$ & & & \\
\hline $\begin{array}{l}\text { Porter, Alan } \\
\text { L. }\end{array}$ & 5 & 3 & Georgia Institute of Technology & Atlanta & EUA \\
\hline $\begin{array}{l}\text { Booyens, } \\
\text { Irma }\end{array}$ & 3 & 2 & $\begin{array}{c}\text { Human Sciences Research } \\
\text { Council }\end{array}$ & $\begin{array}{l}\text { Cidade do } \\
\text { Cabo }\end{array}$ & $\begin{array}{l}\text { África do } \\
\text { Sul }\end{array}$ \\
\hline $\begin{array}{l}\text { Czarnitzki, } \\
\text { Dirk }\end{array}$ & 2 & 2 & $\begin{array}{c}\text { Centre for European Economic } \\
\text { Research }(Z E W)\end{array}$ & Mannheim & Alemanha \\
\hline $\begin{array}{l}\text { Mahroum, } \\
\text { Sami }\end{array}$ & 2 & 2 & INSEAD & Abu Dhabi & $\begin{array}{c}\text { Emirados } \\
\text { Árabes }\end{array}$ \\
\hline $\begin{array}{c}\text { Crisan, } \\
\text { Daniela A. }\end{array}$ & 2 & 2 & Romanian-American University & Bucareste & Romênia \\
\hline $\begin{array}{l}\text { Liebreich, } \\
\text { Michael }\end{array}$ & 2 & 2 & Bloomberg New Energy Finance & Nova York & EUA \\
\hline $\begin{array}{l}\text { Blankley, } \\
\text { William O. }\end{array}$ & 2 & 1 & $\begin{array}{c}\text { Human Sciences Research } \\
\text { Council }\end{array}$ & $\begin{array}{l}\text { Cidade do } \\
\text { Cabo }\end{array}$ & $\begin{array}{l}\text { África do } \\
\text { Sul }\end{array}$ \\
\hline $\begin{array}{l}\text { Steel, G. } \\
\text { Daniel }\end{array}$ & 2 & 1 & Lincoln University & Lincoln & $\begin{array}{c}\text { Nova } \\
\text { Zelândia }\end{array}$ \\
\hline $\begin{array}{l}\text { Schmoch, } \\
\text { Ulrich }\end{array}$ & 2 & 1 & $\begin{array}{c}\text { Fraunhofer Institute for Systems } \\
\text { and Innovation Research }\end{array}$ & Karlshure & Alemanha \\
\hline $\begin{array}{l}\text { Preda, Ana- } \\
\text { Maria }\end{array}$ & 2 & 1 & Romanian-American University & Bucareste & Romênia \\
\hline $\begin{array}{l}\text { Rinne, } \\
\text { Tiffany }\end{array}$ & 2 & 1 & Lincoln University & Lincoln & $\begin{array}{c}\text { Nova } \\
\text { Zelândia }\end{array}$ \\
\hline
\end{tabular}

Fonte: Elaborado pelos autores

O grupo de pesquisa mais representativo em termos de publicações é formado por Alan L.

Porter, que é autor de três trabalhos e coautor de dois (Porter, 2005, 2007a, 2007b; Watts \& Porter, 
Rafael de Moura Speroni, Gertrudes Aparecida Dandolini, João Artur Souza \& Fernando A. Ostuni Gauthier

2003; Zhu \& Porter, 2002). Embora seja o grupo com mais publicações, no conjunto pesquisado, é composto por apenas três pesquisadores, oriundos de três instituições diferentes. Alan L Porter é professor emérito da School of Public Policy e do Industrial \& Systems Engineering (ISyE). Sua maior concentração é avaliação e previsão tecnológica, é codiretor do Technology Policy and Assessment Center e é diretor de P\&D no Search Technology Inc.

Irma Booyens aparece como autora de dois trabalhos e coautora de mais um (Blankley \& Booyens, 2010; Booyens, 2011; Booyens, Molotja, \& Phiri, 2013). Este grupo é composto por quatro pesquisadores, todos vinculados ao Human Sciences Research Council. Irma Booyens desenvolveu pesquisas sobre estudos de viabilidade, de impacto econômico, plano de negócios e estratégias de desenvolvimento do turismo, tendo sido pesquisadora sênior da instituição, e desenvolve, atualmente, pesquisas sobre inovação e networking na indústria do turismo.

Dirk Czarnitski é pesquisador do Centre for European Economic Research e é autor de dois trabalhos (Czarnitzki, Hanel, \& Rosa, 2011; Czarnitzki \& Kraft, 2004), formando um grupo composto por mais três pesquisadores, oriundos de três diferentes instituições. Czarnitski é professor associado da Managerial Economics, Strategy and Innovation, na Katholieke Universiteit Leuven, e é pesquisador sênior na Steunpt $O \& O$ Indicatoren (SOOI). Suas pesquisas têm interesse principalmente no campo de economia da inovação, com foco em microeconometria aplicada, em tópicos como avaliação de políticas públicas de inovação, inovação e governança coorporativa, avaliação de capital intelectual e transferência de tecnologia e conhecimento.

Sami Mahroum, autor de dois artigos (Mahroum \& Alsaleh, 2012, 2013), é diretor acadêmico e administrativo da INSEAD's Innovation and Policy Initiative, em Abu Dhabi. Mahroum foi, anteriormente, analista sênior da OECD, e diretor de pesquisa da National Endowment for Science, Technology and Arts (NESTA). Tem publicações em vários temas relacionados a políticas, como a mensuração da performance de inovação das nações, o brain-drain (emigração de indivíduos com conhecimentos ou capacidades técnicas das organizações, indústrias ou regiões) e a concorrência internacional por talentos.

Daniela A.Crişan é autora de dois artigos (Crişan, Preda, Coculescu, \& AltAr-Samuel, 2010; Preda, Stanica, Crisan, \& Coculescu, 2011), e Ana-Maria Preda, autora de um artigo. Juntas, fazem parte de um grupo com mais três pesquisadores, todos vinculados à Romanian-American University. Crişan é full associate professor e secretária científica desta instituição. Tem competências nas áreas de modelagem matemática, linguagens de programação, engenharia de software e sistemas, além de ter publicado oito livros e mais de sessenta artigos científicos em journals e conferências. Preda é professora na mesma instituição.

Revista de Administração e Inovação, São Paulo, v. 12, n.4 p. 49-75, out./dez. 2015. 
Michael Liebreich é autor único de dois artigos (Liebreich, 2005, 2006), e é o CEO da Bloomberg New Energy Finance, companhia fundada por ele em 2004, e adquirida pela Bloomberg em 2009. Liebreich é membro de vários grupos industriais, incluindo o World Economic Forum's Global Agenda Council in the New Energy Architecture, Accenture's Global Energy Board no grupo consultivo de Energia e Mudanças Climáticas da Secretaria Geral das Nações Unidas. É, também, professor visitante no London's Imperial College Energy Futures Lab e membro do Painel Consultivo do INSEAD Energy Club.

William Blankley é autor de um artigo e coautor de outro (Blankley \& Booyens, 2010; Moses et al., 2012), em um grupo contendo outros seis pesquisadores, todos vinculados ao Centre for Science, Technology and Innovation Indicators (CeSTII), no Human Sciences Research Council, da África do Sul. Blankley é diretor deste centro de pesquisa, e trabalha com análise de políticas de ciência e tecnologia, indicadores de ciência e tecnologia, pesquisa e desenvolvimento e surveys de inovação, planejamento estratégico, estudos de viabilidade e entendimento público da ciência.

Daniel G. Steel é autor de um e coautor de um artigo (Rinne, Steel, \& Fairweather, 2012; Steel, Rinne, \& Fairweather, 2012), publicados com outros dois pesquisadores, todos vinculados à Lincoln University, na Nova Zelândia. Steel é chefe do Department of Social Science, Parks, Recreation, Tourism and Sport da instituição e realiza pesquisas sobre Adaptação psicológica a ambientes extremos e não usuais, redes sociais, inovação e personalidade. Tendo autoria nestes mesmos artigos, Tiffany Rinne trabalha na produção de modelos de cultura, identidade nacional e inovação. O objetivo de sua pesquisa é compreender quais elementos culturais ajudam e quais atrapalham na inovação na Nova Zelândia.

Ulrich Schmoch, autor de um artigo e coautor de outro (Frietsch \& Schmoch, 2010; Schmoch \& Gauch, 2009), é gestor de projetos no Fraunhofer ISI, chefe do programa de Mestrado em Ciência e Gestão da Pesquisa. Schmoch tem numerosas publicações, especialmente nas áreas de indicadores de inovação e interação de ciência e tecnologia.

A

Revista de Administração e Inovação, São Paulo, v. 12, n.4 p. 49-75, out./dez. 2015. 
Tabela 5 ilustra as instituições mais representativas em termos de quantidade de autores a elas vinculados que pesquisam assuntos relacionados com Indicadores de Inovação. 
Tabela 5 - Quantidade de autores por instituição

\begin{tabular}{lc}
\hline Instituição & $\begin{array}{c}\text { Quantidade de } \\
\text { Autores }\end{array}$ \\
\hline Human Sciences Research Council & 9 \\
Dalian University of Technology & 7 \\
Fraunhofer Institute for Systems and Innovation Research & 6 \\
Universidad Politécnica de Valencia & 6 \\
Universidade Federal de São Carlos & 5 \\
Romanian-American University & 5 \\
Universidad Pontifícia Bolivariana & 5 \\
\hline
\end{tabular}

Fonte: Elaborado pelos autores

A instituição com maior número de autores publicando sobre o tema indicadores de inovação é o Human Sciences Research Council, com nove autores. Seu principal objetivo é a condução de projetos sóciocientíficos de grande escala e relevantes politicamente para usuários do setor público, organizações não governamentais e agências de desenvolvimento internacional. $O$ centro de pesquisa ao qual os autores estão vinculados é o Centre for Science, Technology and Innovation Indicators (CeSTII), cujo estabelecimento reflete o movimento global das economias para uma orientação mais intensiva em conhecimento.

O CeSTII foi estabelecido para encarregar-se do desenvolvimento de surveys nacionais e regulares de P\&D e inovação, em nome do Departamento de Ciência e Tecnologia (DST) da África do Sul, e produzir indicadores nacionais de inovação a partir dos resultados, provendo subsídios para políticas e servindo como base para comparações internacionais.

A Dalian University of Technology é uma instituição chinesa, onde destaca-se o WISELAB (Webometrics, Informetrics, Scientometrics and Econometrics Lab), da School of Humanities and Social Science DUT. O WISELAB desenvolve projetos com interesse em Estrutura e Distribuição de Ciência e Tecnologia, História da Ciência e Tecnologia e Pensamento Ético, Sistemas de Inovação e Modelos de Inovação.

O Fraunhofer Institute for Systems and Innovation Researché um instituto de pesquisa que analisa as origens e impactos das inovações. São pesquisados os desenvolvimentos de curto e longo prazo do processo de inovação e os impactos das novas tecnologias e serviços na sociedade. Dentre suas áreas de atuação citam-se: análises comparativas de sistemas de inovação em nível nacional, setorial e tecnológico; previsão de tecnologias e criação de cenários e roteiros para futuros desenvolvimentos tecnológicos; investigação dos contextos institucional e regulatório da inovação; análise do processo de difusão da inovação; avaliação da inovação e seus potenciais em uma perspectiva econômica, social e ecológica; estimativa e opções de políticas relacionadas à inovação,

Revista de Administração e Inovação, São Paulo, v. 12, n.4 p. 49-75, out./dez. 2015. 
Rafael de Moura Speroni, Gertrudes Aparecida Dandolini, João Artur Souza \& Fernando A. Ostuni Gauthier

bem como as chances de sucesso e aceitação no mercado e sociedade, e; aconselhamento de players industriais e criadores de políticas na introdução e implementação de soluções inovadoras.

Na Universidad Politécnica de Valencia destacam-se os autores vinculados ao Instituto de Gestión de la Innovación y del Conocimiento, INGENIO, que é um instituto de pesquisa em conjunto com o Spanish National Council of Scientific Research. Dentre as atividades desenvolvidas pelo INGENIO, estão as pesquisas em ciência e inovação, com abordagens sobre a emergência e evolução da tecnologia, processos organizacionais que facilitam seu desenvolvimento, e políticas que a suportam.

A Universidade Federal de São Carlos conta com cinco publicações, sendo quatro delas de autoria de pesquisadores vinculados ao Departamento de Engenharia de Materiais, que é um órgão acadêmico de geração e desenvolvimento de atividades interdisciplinares de ensino, pesquisa científica e desenvolvimento tecnológico. O departamento conta com grupos interdisciplinares, atuando em ensino teórico, experimental de campo, estágios de treinamento, interação com indústrias, participação em órgãos de fomento, entidades representativas e em congressos nacionais e internacionais.

As universidades Romanian-American University, Universidad Pontifícia Bolivariana também contam com cinco publicações. A primeira, localizada em Bucareste, conta com seis faculdades em sua estrutura, sendo cinco deles do setor econômico e um jurídico. A segunda, na cidade de Medellin, é uma instituição de ensino superior privada e conta com um Centro de Pesquisa para o Desenvolvimento e Inovação (CIDI), que integra os trabalhos dos grupos de pesquisa e das unidades de transferência de conhecimento para transformar em riqueza.

\subsection{Principais palavras-chave e temáticas abordadas pelos trabalhos}

Os trabalhos analisados utilizaram 300 palavras-chave. As com maior número de ocorrências foram: innovation, com 34 ocorrências; innovation indicators, com 12 ocorrências; indicators e technological innovation, com oito ocorrências cada; innovation index e patents, com sete ocorrências cada, e; composite indicators e research and development, com cinco ocorrências cada.

A Erro! Fonte de referência não encontrada. apresenta a nuvem de termos construída com as palavras-chave constantes nos trabalhos, onde o tamanho da fonte na figura representa a intensidade de ocorrência. Cabe salientar que os termos "innovation indicators" e "innovation index" são os termos de busca e, por isso, procedeu-se a filtragem das palavras-chave, visando remover aquelas cujo destaque seria óbvio, possibilitando a melhor visualização dos demais

Revista de Administração e Inovação, São Paulo, v. 12, n.4 p. 49-75, out./dez. 2015. 
termos associados às palavras-chave. Foram removidos, ainda, nesta fase, os termos "innovation", "indicators", "technological innovation".

Figura 3 - Representação de ocorrência das palavras-chave após remoção de termos

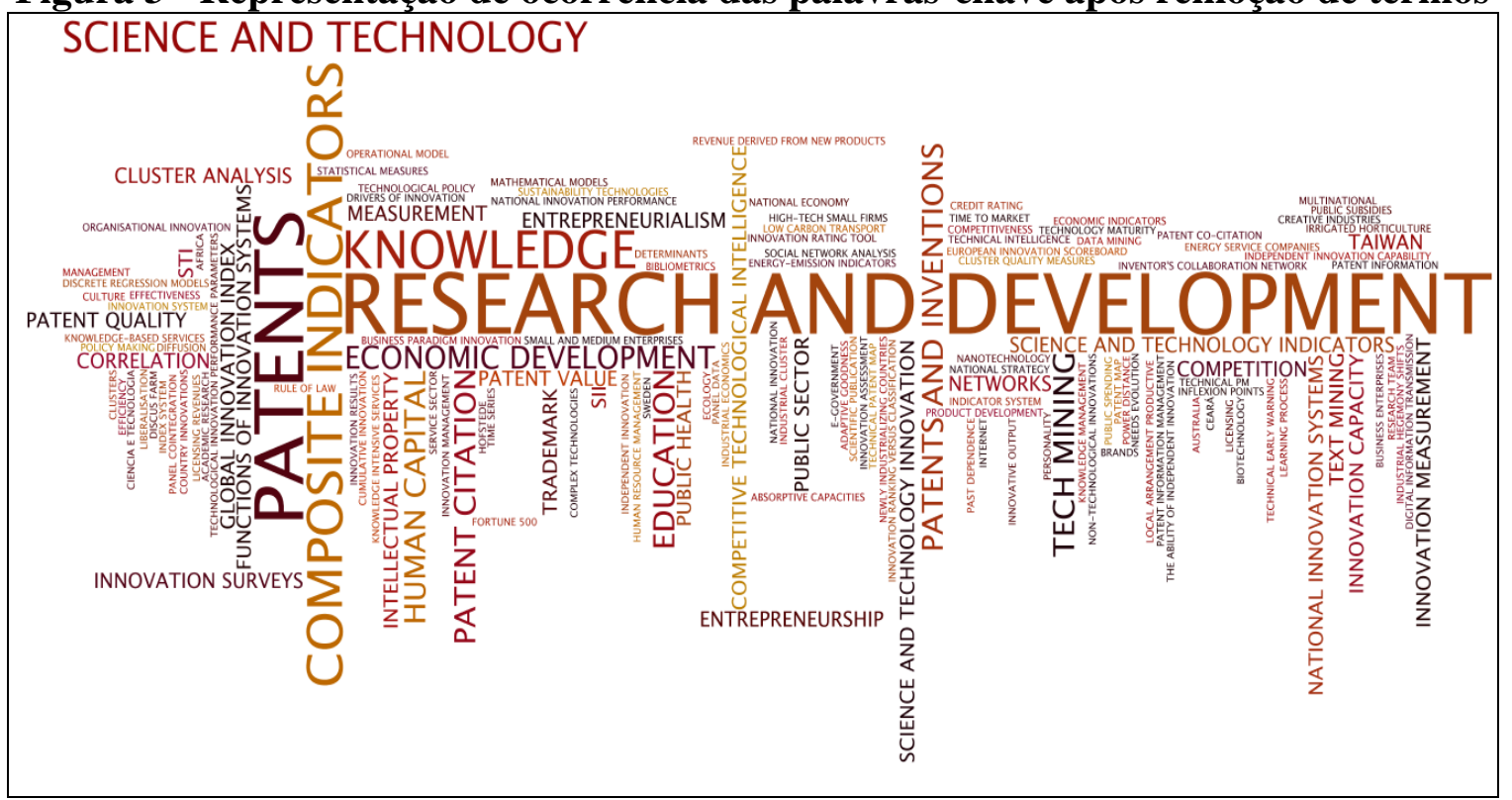

Fonte: Elaborado pelos autores

$\mathrm{Na}$ Erro! Fonte de referência não encontrada. é possível observar que recebem destaque considerável os termos "research and development", "patents", "composite indicators", "knowledge" e "science and technology". Ainda associados ao termo "patents", observam-se, em menor destaque, "patent citation", "patents and inventions", "patent value","patent quality" e "patent co-citation". Associado ao termo "science and technology", tem-se os termos "science and technology innovation" e "STI". O termo "composite indicators" tem relação com "innovation surveys", uma vez que este último trata de iniciativas de pesquisas que adotam índices compostos de inovação para traçar comparativos entre regiões e/ou países.

O destaque a termos associados com patentes (patents) (Baron \& Delcamp, 2012; Frietsch \& Schmoch, 2010; Makkonen \& van der Have, 2013; Wang, Zhang, \& Xu, 2011), pesquisa e desenvolvimento (research and development) (Czarnitzki et al., 2011; Porter, 2007a; Watts \& Porter, 2003), e indicadores compostos (composite indicators) (Grupp \& Schubert, 2010; Mahroum \& Alsaleh, 2012, 2013; Paas \& Poltimäe, 2012) justifica-se pelo fato de que, tradicionalmente, os dados associados aos dois primeiros termos são utilizados como principais indicadores de inovação. Mais recentemente, entretanto, diversas iniciativas passaram a adotar o uso de indicadores compostos.

Corroborando com a observação, Makkonen and van der Have (2013), em sua revisão de literatura, lembram que o tipo de medida escolhida para mensurar a inovação pode afetar substancialmente as conclusões das pesquisas ou as informações nas quais os tomadores de decisão se 
Rafael de Moura Speroni, Gertrudes Aparecida Dandolini, João Artur Souza \& Fernando A. Ostuni Gauthier

baseiam para a criação de políticas. Isto fez com que diferentes medidas fossem adotadas ao longo do tempo, iniciando com o uso de estatísticas de patentes, a partir dos anos 1950, seguido pelos investimentos em P\&D, em meados dos anos 1980 e pela adoção de uma conceitualização mais ampla da inovação em surveys a partir dos anos 1990.

O entendimento de que a inovação é vista como um processo, compreendendo, portanto, outros aspectos das organizações, bem como de variáveis externas e que levem em conta o ambiente onde estão inseridas, reflete-se na ocorrência de termos como "knowledge" (Blankley \& Booyens, 2010; Hollenstein, 2003; Porter, 2005; Rongdi \& Jun, 2011; Schmoch \& Gauch, 2009), "human capital" (de Brito Cruz \& Mello, 2006; Gates \& Langevin, 2010; Joiozo \& Teles, 2011), "intellectual property" (Czarnitzki \& Kraft, 2004; Gotsch \& Hipp, 2012; INSEAD \& Karabell, 2012) e "education" (Johnson \& Chuang, 2010; Joiozo \& Teles, 2011; Leydesdorff, 2004; Muresan \& Gogu, 2012), que sugerem abordagens onde o fator humano e o conhecimento são levados em consideração na mensuração da inovação.

\subsection{Referências mais citadas}

Como embasamento teórico, os artigos analisados utilizaram 2.583 referências, o que representa uma média de aproximadamente 24 referências por trabalho. A Tabela 6 resume as referências mais utilizadas pelos autores, as quais, em seguida, são descritas individualmente.

A publicação mais citada, com 14 ocorrências, foi o Oslo Manual - Proposed guidelines for collecting and interpreting innovation data, publicado em 2005 pela Organisation for Economic Cooperation and Development (OECD). Trata-se da terceira edição de um manual que tem por objetivo a orientar e padronizar conceitos, metodologias e construção de estatísticas e indicadores de pesquisa de P\&D de países industrializados.

A segunda maior ocorrência foi o Frascati Manual 2002: Proposed Standard Practice for Surveys on Research and Experimental Development, referenciado por 12 publicações. O Manual de Frascati é uma proposta que contem as definições básicas e categorias das atividades de P\&D, tendo sido aceitas por pesquisadores do mundo todo. Assim, a publicação é reconhecida como uma referência para determinar quais atividades são consideradas de P\&D. A versão do ano de 2002 é a sexta edição do documento, e tem como propósito apontar as tendências de globalização em ciências, tecnologias e economias em relação à $\mathrm{P} \& \mathrm{D}$.

Revista de Administração e Inovação, São Paulo, v. 12, n.4 p. 49-75, out./dez. 2015. 
Estado da arte da produção científica sobre indicadores e índices de inovação

Tabela 6 - As dez referências mais utilizadas pelos trabalhos analisados

\begin{tabular}{|c|c|c|c|c|c|}
\hline & Autores & Ano & Título & Fonte de publicação & Qtd \\
\hline 1 & OECD & 2005 & $\begin{array}{l}\text { Oslo Manual - Proposed } \\
\text { guidelines for collecting and } \\
\text { interpreting innovation data }\end{array}$ & OECD & 14 \\
\hline 2 & OECD & 2002 & $\begin{array}{c}\text { Frascati Manual 2002: Proposed } \\
\text { Standard Practice for Surveys on } \\
\text { Research and Experimental } \\
\text { Development }\end{array}$ & OECD & 12 \\
\hline 3 & Lundvall, B-A. & 1992 & $\begin{array}{l}\text { National Innovation Systems: } \\
\text { Towards a Theory of Innovation } \\
\text { and Interactive Learning }\end{array}$ & Pinter & 09 \\
\hline 4 & $\begin{array}{l}\text { Schumpeter, } \\
\text { J.A. }\end{array}$ & 1934 & $\begin{array}{c}\text { The Theory of Economic } \\
\text { Development - An Inquiry into } \\
\text { Profits, Capital, Credit, Interest, } \\
\text { and the Business Cycle }\end{array}$ & Harward University Press & 09 \\
\hline 5 & Griliches, Z. & 1990 & $\begin{array}{c}\text { Patent Statistics as Economic } \\
\text { Indicators: A Survey }\end{array}$ & $\begin{array}{c}\text { Journal of Economic } \\
\text { Literature }\end{array}$ & 08 \\
\hline 6 & Nelson, R.R. & 1993 & $\begin{array}{c}\text { National Innovation Systems: A } \\
\text { Comparative Analysis }\end{array}$ & Oxford University Press & 08 \\
\hline 7 & $\begin{array}{l}\text { Schumpeter, } \\
\text { J.A. }\end{array}$ & 1943 & $\begin{array}{c}\text { Capitalism, Socialism and } \\
\text { Democracy }\end{array}$ & Harper \& Brothers & 08 \\
\hline 8 & $\begin{array}{l}\text { Cohen, W.M.; } \\
\text { Levinthal, D.A. }\end{array}$ & 1990 & $\begin{array}{l}\text { Absorptive capacity: a new } \\
\text { perspective on learning and } \\
\text { innovation }\end{array}$ & $\begin{array}{c}\text { Administrative Science } \\
\text { Quarterly }\end{array}$ & 07 \\
\hline 9 & Smith, K & 2005 & Measuring Innovation & $\begin{array}{l}\text { Handbook of Innovation. } \\
\text { Oxford University Press }\end{array}$ & 07 \\
\hline
\end{tabular}

Fonte: Elaborado pelos autores

O livro National Innovation Systems: Towards a Theory of Innovation and Interactive Learning, publicado em 1992, por Bengt-Åke Lundval, aparece como o terceiro mais referenciado, com nove ocorrências. Esse livro aborda a dinâmica da economia nacional e global, e parte do princípio de que a competição internacional entre as nações é baseada na inovação. Busca investigar o papel desempenhado por cada uma das diferentes partes do sistema nacional na determinação das dinâmicas de longo prazo da economia, bem como identificar o que acontece com a coerência dos sistemas nacionais de inovação em uma era caracterizada pela internacionalização de longo alcance e globalização.

Outra obra que aparece com nove ocorrências é o livro The Theory of Economic Development An Inquiry into Profits, Capital, Credit, Interest, and the Business Cycle, de Joseph Alois Schumpeter, um clássico de 1934 que faz uma análise da sociedade capitalista e sua economia. Schumpeter apresenta teorias que são baseadas na lógica e fornecem estrutura para a compreensão.

No artigo Patent Statistics as Economic Indicator: A Survey, de Zvi Griliches, publicado em 1990, com oito ocorrências, o autor trata do uso de estatísticas de patentes. No artigo Griliches 
Rafael de Moura Speroni, Gertrudes Aparecida Dandolini, João Artur Souza \& Fernando A. Ostuni Gauthier

descreve a natureza das patentes e os tipos de dados gerados, sua disponibilidade e os principais problemas que surgem quando se tenta usá-los em análises econômicas. Faz ainda uma revisão sobre os trabalhos correlatos, e aborda a questões como os possíveis usos de estatísticas de patentes, e tenta responder questões levantadas por trabalhos anteriores de outros autores.

O livro National Innovation Systems: A Comparative Analysis, de Richard R. Nelson, com oito ocorrências, foi publicado em 1993, e parte de um princípio chamado "tecno-nacionalismo", força tecnológica observada em países como o Japão, Taiwan e Korea nos anos 70 e 80, que combina a crença de que as capacidades tecnológicas de uma nação são fatores chave para o processo competitivo, com a crença segundo a qual tais capacidades existem em nível nacional, e podem ser construídas por ações nacionais. O livro trata de sistemas nacionais de inovação e contem estudos sobre dezessete países, destacando mecanismos e instituições que suportam a inovação, e apresentando similaridades e diferenças entre os países.

Também com oito ocorrência, o livro Capitalism, Socialism and Democracy, de Joseph Alois Schumpeter, publicado em 1943, é considerado um clássico da teoria social. Nesse livro Schumpeter afirma que as causas do declínio do capitalismo eram internas, e mostra uma oposição ao socialismo centralista.

Com sete ocorrências, no artigo Absorptive capacity: a new perspective on learning and innovation, de Welsey M. Cohen, publicado em 1990, o autor argumenta que a habilidade de uma empresa em reconhecer o valor das informações novas e externas, assimilá-las e aplicá-las para fins comerciais é crítica para suas capacidades inovadoras, e sugere que esta capacidade absortiva é uma função do seu nível de conhecimento prévio. O artigo foca nas bases cognitivas para uma capacidade absortiva individual, e caracteriza os fatores que influenciam a capacidade absortiva em nível organizacional.

Finalmente, também com sete ocorrências, o artigo Measuring Innovation, de Keith Smith, é um capítulo do livro The Oxford Handbook of Innovation, publicado em 2005. Neste capítulo, o autor argumenta que, embora a afirmação de alguns autores que consideram a inovação inerentemente impossível de quantificar e medir possa ser verdadeira em alguns aspectos, as características da inovação não impedem a mensuração de dimensões-chave de processos e saídas. Aborda a emergência de indicadores de inovação de entrada e saída, e a discussão de algumas questões na construção e uso de indicadores de ciência, tecnologia e inovação, como as potencialidades e fragilidades dos indicadores utilizados naquele momento, particularmente $\mathrm{P} \& \mathrm{D}$ e patentes.

Revista de Administração e Inovação, São Paulo, v. 12, n.4 p. 49-75, out./dez. 2015. 


\section{CONSIDERAÇÕES FINAIS}

Neste artigo foram apresentados os resultados de uma pesquisa bibliométrica feita usando os termos de busca "innovation indicator" e "innovation index". Utilizando técnicas bibliométricas de recuperação de informação, o trabalho traz um mapeamento sobre o perfil e as características das pesquisas que tratam do assunto referente aos termos buscados em quatro bases dados científicas.

A análise dos dados demonstra que, embora o tema não seja novo, visto que a primeira publicação encontrada é do ano de 1973, a maior ocorrência de publicações acontece a partir do ano de 2009, situação que permanece até o ano de 2013, ainda que os dados desse ano sejam parciais, uma vez que a busca foi realizada no mês de outubro.

A distribuição geográfica dos autores indica maiores concentrações no oeste europeu e na América do Norte. Embora haja certos pontos de concentração geográfica dos autores, observa-se pouca inter-relação entre os autores de diferentes locais, fazendo com que não se observe a existência de grupos de coautoria significativos.

A análise das palavras-chave aponta termos associados à escolha de indicadores de inovação adotados na literatura. Observou-se a relevância dos termos relativos às estatísticas de Ciência e Tecnologia, Patentes e P\&D, comumente utilizados como indicadores individuais. Além destes, também há ocorrência de trabalhos que utilizam a abordagem de indicadores compostos para mensurar a inovação. Este tipo de abordagem considera que há um maior conjunto de variáveis que podem ser observadas, considerando diferentes aspectos internos e externos às organizações e que influenciam nas atividades inovação. Tais trabalhos costumam apresentar modelos onde são utilizadas técnicas estatísticas, tais como análise fatorial e análise de componentes principais para identificação de variáveis sem correlação.

A tendência de utilização de indicadores compostos se confirma na análise de referências mais citadas pelos trabalhos selecionados, que aponta que grande participação de dois trabalhos da OECD (Organisation for Economic Co-operation and Development), especificamente o Manual de Oslo e o Manual de Frascati. Tais documentos apresentam guias para a mensuração da inovação especialmente em nível nacional, e apontam para a construção de Índices Compostos de Inovação.

Para trabalhos futuros, recomenda-se a pesquisa em outras bases de dados incluindo as nacionais, como a Scielo. Sugere-se, também, a ampliação da abrangência da busca, com a utilização de outros termos de busca, como "innovation measuring" e "innovation assessment", com o objetivo de tornar a busca mais abrangente.

Revista de Administração e Inovação, São Paulo, v. 12, n.4 p. 49-75, out./dez. 2015. 
Rafael de Moura Speroni, Gertrudes Aparecida Dandolini, João Artur Souza \& Fernando A. Ostuni Gauthier

Ainda como sugestão de trabalho futuro, verificar a possibilidade de um estudo comparativo entre os diferentes modelos de indicadores de inovação compostos, visando identificar as características e estruturas em comum entre eles.

\section{REFERÊNCIAS}

Araújo, R. F. d., \& Alvarenga, L. (2011). A Bibliometria na Pesquisa Científica da Pós-Graduação Brasileira de 1987 a 2007. Revista Eletrônica de Biblioteconomia e Ciência da Informação, 16(31). doi: 10.5007/1518-2924.2011V16N31P51

Baron, J., \& Delcamp, H. (2012). The private and social value of patents in discrete and cumulative innovation. Scientometrics, 90(2), 581-606. doi: 10.1007/s11192-011-0532-5

Bessant, J., \& Tidd, J. (2009). Inovação e Empreendedorismo. Porto Alegre: Bookman.

Blackman Jr, A. W., Seligman, E. J., \& Sogliero, G. C. (1973). An innovation index based on factor analysis. Technological Forecasting and Social Change, 4(3), 301-316.

Blankley, W. O., \& Booyens, I. (2010). Building a Knowledge Economy in South Africa. South African Journal of Science, 106(11/12), 15-20. doi: 10.4102/sajs.v106i11/12.373

Booyens, I. (2011). Are small, medium- and micro-sized enterprises engines of innovation? The reality in South Africa. Science \& Public Policy (SPP), 38(1), 67-78. doi: $10.3152 / 030234211 \mathrm{X} 12834251302445$

Booyens, I., Molotja, N., \& Phiri, M. (2013). Innovation in High-Technology SMMEs: The Case of the New Media Sector in Cape Town. Urban Forum, 24(2), 289-306. doi: 10.1007/s12132-012-9168-7

Cornell University, INSEAD, \& WIPO. (2013). The Global Innovation Index 2013: The Local Dynamics of Innovation. Geneva, Switzerland.

Crişan, D. A., Preda, A.-M., Coculescu, C., \& AltAr-Samuel, A. N. (2010). Some Aspects Concerning the Correlation Between ICT and Innovation in Europe. Annals of the University of Oradea, Economic Science Series, 19(2), 1183-1189.

Czarnitzki, D., Hanel, P., \& Rosa, J. M. (2011). Evaluating the impact of R\&D tax credits on innovation: A microeconometric study on Canadian firms. Research Policy, 40(2), 217-229. doi: 10.1016/j.respol.2010.09.017

Czarnitzki, D., \& Kraft, K. (2004). Innovation indicators and corporate credit ratings: Evidence from German firms. Economics Letters, 82(3), 377-384. doi: 10.1016/j.econlet.2003.09.016

de Brito Cruz, C. H., \& Mello, L. d. (2006). Boosting Innovation Performance in Brazil. Stimuler l'innovation en Brésil., 6(13), 1-33. doi: 10.1787/357276015553 
Frietsch, R., \& Schmoch, U. (2010). Transnational patents and international markets. Scientometrics, 82(1), 185-200. doi: 10.1007/s11192-009-0082-2

Gates, S., \& Langevin, P. (2010). Human capital measures, strategy, and performance: HR managers' perceptions. Accounting, Auditing and Accountability Journal, 23(1), 111-132. doi: $10.1108 / 09513571011010628$

Gotsch, M., \& Hipp, C. (2012). Measurement of innovation activities in the knowledge-intensive services industry: a trademark approach. Service Industries Journal, 32(13), 2167-2184. doi: $10.1080 / 02642069.2011 .574275$

Grupp, H., \& Schubert, T. (2010). Review and new evidence on composite innovation indicators for evaluating national performance. Research Policy, 39(1), 67-78. doi: 10.1016/j.respol.2009.10.002

Hollenstein, H. (2003). Innovation modes in the Swiss service sector: A cluster analysis based on firmlevel data. Research Policy, 32(5), 845-863. doi: 10.1016/S0048-7333(02)00091-4

IBGE, C. d. I. (2010). Pesquisa de inovação tecnológica: 2008. Rio de Janeiro: IBGE.

INSEAD, \& Karabell, S. (2012). Global Innovation Index 2012: Stronger innovation linkages for global growth INSEAD Knowledge Publications (pp. 1-2): INSEAD.

Johnson, W. H. A., \& Chuang, M. (2010). A comparative innovation study of China, Japan and Taiwan. Chinese Management Studies, 4(4), 385-400. doi: 10.1108/17506141011094154

Joiozo, R., \& Teles, V. K. (2011). Human capital and innovation: Evidence from panel cointegration tests. Applied Economics Letters, 18(17), 1629-1632. doi: 10.1080/13504851.2011.556584

Kleinknecht, A., Van Montfort, K., \& Brouwer, E. (2002). The Non-Trivial Choice Between Innovation Indicators. Economics of Innovation \& New Technology, 11(2), 109.

Kobashi, N. Y., \& Santos, R. N. M. d. (2006). Institucionalização da pesquisa científica no Brasil:cartografia temática e de redes sociaispor meio de técnicas bibliométricas. TranInformação, 18(1), 10.

Leydesdorff, L. (2004). The university-industry knowledge relationship: Analyzing patents and the science base of technologies. Journal of the American Society for Information Science and Technology, 55(11), 991-1001. doi: 10.1002/asi.20045

Liebreich, M. (2005). GEIX market capitalisation doubles in first three quarters of 2005. Refocus, 6(6), 16-17. doi: 10.1016/S1471-0846(05)70481-9

Liebreich, M. (2006). The WilderHill New Energy Global Innovation Index (NEX). Refocus, 7(4), 2223. doi: 10.1016/S1471-0846(06)70616-3

Macias-Chapula, C. A. (1998). O papel da informetria e da cienciometria e sua perspectiva nacional e internacional. Ciência da Informação, 27(2), 7.

Mahroum, S., \& Alsaleh, Y. (2012). Measuring Innovation Efficacy: An Operational Framework for Mapping and Measuring Innovation Capacity and Performance of Countries INSEAD Working Papers Collection (pp. 1-39): INSEAD. 
Rafael de Moura Speroni, Gertrudes Aparecida Dandolini, João Artur Souza \& Fernando A. Ostuni Gauthier

Mahroum, S., \& Alsaleh, Y. (2013). Towards a functional framework for measuring national innovation efficacy. Technovation. doi: 10.1016/j.technovation.2013.03.013

Makkonen, T., \& Have, R. P. (2013). Benchmarking regional innovative performance: composite measures and direct innovation counts. Scientometrics, 94(1), 247-262. doi: 10.1007/s11192-0120753-2

Makkonen, T., \& van der Have, R. P. (2013). Benchmarking regional innovative performance: Composite measures and direct innovation counts. Scientometrics, 94(1), 247-262. doi: $10.1007 / \mathrm{s} 11192-012-0753-2$

Moses, C., Sithole, M. M., Blankley, W., Labadarios, D., Makelane, H., \& Nkobole, N. (2012). The state of innovation in South Africa: Findings from the South African National Innovation Survey. South African Journal of Science, 108(7/8), 1-5. doi: 10.4102/sajs.v108i7/8.1320

Muresan, M., \& Gogu, E. (2012). Tertiary education's role in research and innovation. Paper presented at the 4th World Conference on Educational Sciences.

Nelson, A. (2009). Measuring knowledge spillovers: What patents, licenses and publications reveal about innovation diffusion. Research Policy, 38(6), 12.

OECD. (2005). Oslo Manual - Guidelines for Collecting and Interpreting Innovation Data (3 ed.). Paris, France: OECD.

Paas, T., \& Poltimäe, H. (2012). Consistency between innovation indicators and national innovation performance in the case of small economies. Eastern Journal of European Studies, 13(1), 101-121.

Pizzani, L. e. a. (2012). A arte da pesquisa bibliográfica na busca do conhecimento. Rev. Dig. Bibl. Ci. Inf, 10(1), 53-66.

Porter, A. L. (2005). QTIP: Quick technology intelligence processes. Technological Forecasting and Social Change, 72(9), 1070-1081. doi: 10.1016/j.techfore.2004.10.007

Porter, A. L. (2007a). How "tech mining" can enhance R\&D management. Research Technology Management, 50(2), 15-20.

Porter, A. L. (2007b). Tech mining to accelerate radical innovation, Portland, OR.

Preda, A.-M., Stanica, J. L., Crisan, D. A., \& Coculescu, C. (2011). E-Government: A Driving Factor for Stimulating Innovation Performance in Romania? Annals of the University of Oradea, Economic Science Series, 20(1), 125-130.

Quintane, E., Casselman, R. M., Reiche, B. S., \& Nylund, P. A. (2011). Innovation as knowledgebased outcome. Journal of Knowledge Management, 15(6).

Ratanawaraha, A., \& Polenske, K. R. (2007). Measuring the geography of innovation: A literature review. In K. Polenske (Ed.), The economic geography of innovation (pp. 30). Cambridge Cambridge University Press.

Revista de Administração e Inovação, São Paulo, v. 12, n.4 p. 49-75, out./dez. 2015. 
Ribeiro, H. C. M., Cirani, C. B. S., \& Freitas, E. J. (2013). AnÁlise Da ProduÇÃo CientÍfica Da Revista De AdministraÇÃo E InovaÇÃo. Review of Administration and Innovation - RAI, 10(4), 208. doi: 10.5773/rai.v10i4.1139

Rinne, T., Steel, G. D., \& Fairweather, J. (2012). Hofstede and Shane Revisited: The Role of Power Distance and Individualism in National-Level Innovation Success. Cross-Cultural Research, 46(2), 91-108. doi: 10.1177/1069397111423898

Rongdi, J., \& Jun, W. (2011, 13-15 May 2011). Analysis on determinants of knowledge diffusion based on patent citations. Paper presented at the Business Management and Electronic Information (BMEI), 2011 International Conference on.

Saisana, M., Saltelli, A., \& Tarantola, S. (2005). Uncertainty and sensitivity analysis techniques as tools for the quality assessment of composite indicators. Journal of the Royal Statistical Society. Series A, $168(2), 17$.

Schmoch, U., \& Gauch, S. (2009). Service marks as indicators for innovation in knowledge-based services. Research Evaluation, 18(4), 323-335. doi: 10.3152/095820209X451023

Steel, G. D., Rinne, T., \& Fairweather, J. (2012). Personality, nations, and innovation: Relationships between personality traits and national innovation scores. Cross-Cultural Research, 46(1), 3-30. doi: $10.1177 / 1069397111409124$

Wang, X., Zhang, X. Z., \& Xu, S. (2011). Patent co-citation networks of Fortune 500 companies. Scientometrics, 88(3), 761-770. doi: 10.1007/s11192-011-0414-X

Watts, R. J., \& Porter, A. L. (2003). R and D cluster quality measures and technology maturity. Technological Forecasting and Social Change, 70(8), 735-758. doi: 10.1016/S0040-1625(02)00355-4

Zabala-Iturriagagoitia, J. M., Jiménez-Sáez, F., Castro-Martínez, E., \& Gutiérrez-Gracia, A. (2007). What indicators do (or do not) tell us about Regional Innovation Systems. Scientometrics, 70(1), 85106. doi: 10.1007/s11192-007-0106-8

Zhu, D., \& Porter, A. L. (2002). Automated extraction and visualization of information for technological intelligence and forecasting. Technological Forecasting and Social Change, 69(5), 495506. doi: 10.1016/S0040-1625(01)00157-3 


\title{
STATE OF THE ART OF SCIENTIFIC PRODUCTION ON INDICATORS AND INNOVATION INDEX
}

\begin{abstract}
Innovation management is a challenge for organizations that aim to be competitive. When implementing a management process, the ability to measure what is to be managed is very important as it is the explanation of the strategic model of the innovation. The use of indicators allows verifying the development of the organizations or the development of each one of the regions individually, or in comparison to each other. Identifying indicators for innovation is crucial and there are several tools to help in this process, such as the patent statistics and R\&D. This paper presents a bibliometric review wich aims to map the scientific production about Innovation Indicators. We have analysed 107 scientific articles from 209 authors working for 115 different institutions in 35 countries. Results show that the first article was published in 1973, and the number of publications tends to increase with time. The majority of the articles are published in journals and Europe is the region that counts the largest number of authors, followed by China, Brazil and the United States.
\end{abstract}

Keywords: Innovation Indicator; Innovation Index; Innovation; Bibliometrics; Bibliographic research.

Data do recebimento do artigo: 06/09/2014

Data do aceite de publicação: 05/11/2015

Revista de Administração e Inovação, São Paulo, v. 12, n.4 p. 49-75, out./dez. 2015. 